\title{
THE PARKES-MIT-NRAO RADIO SURVEYS
}

\author{
ALAN E. WRIGHT \\ Parkes Observatory, Australia Telescope National Facility \\ PO Box 276, Parkes, NSW, 2870, Australia \\ NIVEN J. TASKER \\ Macquarie University and the Australia Telescope National Facility \\ PO Box 276, Parkes, NSW, 2870, Australia \\ ANN SAVAGE \\ UK Schmidt Telescope Unit \\ Private Bag, Coonabarabran, NSW 2357, Australia \\ AND \\ ALAN E. VAUGHAN \\ Macquarie University, Dept Physics \& Mathematics, \\ North Ryde, NSW 2113, Australia
}

\section{Introduction}

During 1990, the Parkes radio telescope made a new, deep survey of the southern sky at $4850 \mathrm{MHz}$ (the PMN Survey: see e.g. Griffith and Wright, 1993; Wright et al., 1994). The declination coverage of the survey was from $\delta$ $=-87^{\circ}$ to $+10^{\circ}$. The flux limit of the survey was around $30 \mathrm{mJy}$, although dependent on declination. This survey increased the number of known, southern radio sources by a factor of about 6 to over 65,000 .

From 1992 until the present year, we have been re-observing approximately 8,000 of the stronger southern sources $\left(\mathrm{S}_{4850}>70 \mathrm{mJy}\right.$ and $\delta<$ $-37^{\circ}$ ) using the ATNF Compact Array at Narrabri. These observations were made in order to obtain accurate (s.e. $<1$ arcsec) positions, multi-frequency fluxes, and radio structure.

The third stage of this project was to use these accurate positions, together with the COSMOS digitised sky survey (Yentis et al., 1992), to obtain optical identifications for over 4000 of the objects. We have also 
used the Hubble Space Telescope Guide Star Catalogue for the brighter identifications, and the UK Schmidt plates for the fainter identifications.

The principal result of this project has been a large, homogeneous and complete sample of identified extragalactic radio sources. This database is being used to search for southern gravitational lens candidates, to compile an unbiased list of GPS (Gigahertz Peaked Spectrum) radio sources, and to search for very bright QSO candidates that may have previously escaped detection.

In this paper, we describe some of this work and indicate the initial findings from a subsequent programme of optical spectroscopy of a sample of the identified sources.

\section{The PMN Surveys and Data}

Previous radio surveys of the southern sky have suffered from serious deficiencies. For example, the widely used Parkes $2700 \mathrm{MHz}$ Survey (as contained in the PKSCAT90 database; see Wright and Otrupcek, 1990) does not list sources close to the Galactic Plane and has different flux limits in the different survey zones. This means it can not be easily used for statistical purposes, such as homogeneity tests.

The new PMN survey, taken together with the northern 87GB survey of Jim Condon and colleagues (see e.g. Gregory and Condon, 1991), provides a survey covering the whole sky from $-87^{\circ}<\delta<+75^{\circ}$ made at the same frequency, with the same receiver and multibeam feed system, and with similar resolutions. And, at a level of about $70 \mathrm{mJy}$, it is uniform and essentially complete throughout this declination range. Fig. 1 shows a plot of the PMN sources above this flux limit, where the plot is centred on the south celestial pole and extends northwards to $+10^{\circ}$.

The data from the PMN surveys is available both in the form of point source catalogues (see e.g. Wright et al, 1994) or as a set of images. The simplest way to access this data is from the Australia Telescope's anonymous FTP server as:

ftp.atnf.csiro.au and in the area $/ p u b / d a t a / p m n / C A$.

In addition, the Parkes Observatory's Surveys HomePage on the World Wide Web:

\section{http://wwwpks.atnf.csiro.au/databases/surveys/surveys.html}

presents a useful and "friendly" interface to this server, as well as providing information about the PMN, and other radio surveys.

Besides presenting a much more extensive and uniform catalogue of radio sources, the PMN surveys have permitted much good science to be done. Correlations have been made with existing radio catalogues at other wavelengths, with optical catalogues and with infrared and X-ray cata- 


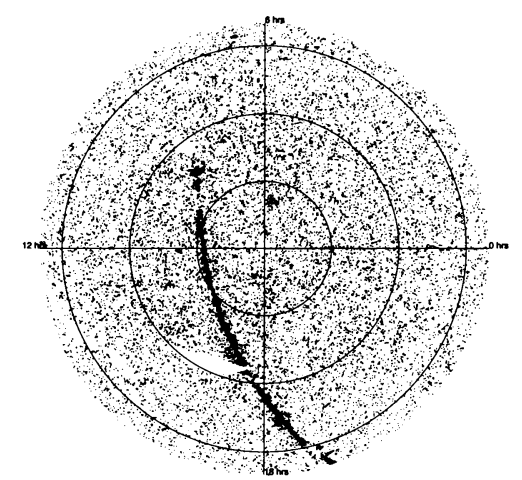

Figure 1. A plot of the stronger sources $\left(\mathrm{S}_{4850}\right.$ greater than $\left.70 \mathrm{mJy}\right)$ found in the various zones of the PMN Surveys. The plot is centred on the South Celestial Pole and right ascension increases in an anti-clockwise direction starting from the right of the plot. The dark band in the figure marks the position of the Galactic Plane. The Large Magellanic Cloud and the strong radio galaxy Cen A can also be distinguished near $(5.5 \mathrm{~h}$, $-70)$ and $(13.5 \mathrm{~h},-40)$ respectively

logues. Furthermore, sensitive tests for anisotropy on the largest scales can be made much more effectively with a deep continuum radio survey than with optical data, which suffers obscuration.

Despite this, however, much more information is revealed about a source once it is optically identified. For example, the redshift is potentially measurable. For this reason, the second stage of our programme attempted to provide optical identifications for as many of the southern $\left(\delta<-37^{\circ}\right)$ radio sources as possible. (A similar, and complementary, programme is being undertaken for the more northerly sources by our collaborators at MIT, B. Burke and A. Fletcher, using the Very Large Array.)

\section{The ATNF Compact Array Programme}

The accuracy of the positions of the sources contained in the point-source catalogues depends on the flux density but is typically around 15 arcsec (standard error) in each coordinate. However, to identify radio sources unambiguously with their optical counterparts, without regard to their optical colour or morphology, positions must be determined with a standard error of less than 2 arcsec - or even more accurately in regions of high star density.

With this in mind, we have undertaken a programme to re-measure the stronger sources contained in the Southern PMN survey. The criteria used to select sources are given in the following table (Table 1).

The observations were made using the Compact Array of the Australia 
TABLE 1. The Compact Array Sample zones

\begin{tabular}{lll}
\hline Declination Range (J2000) & Flux limits & Galactic Latitude \\
\hline$-87^{\circ}<\delta<-73^{\circ}$ & $\mathrm{S}_{4850} \geq 50 \mathrm{mJy}$ & $|b| \geq 2^{\circ}$ \\
$-73^{\circ}<\delta<-38.5^{\circ}$ & $\mathrm{S}_{4850} \geq 70 \mathrm{mJy}$ & $|b| \geq 2^{\circ}$ \\
\hline
\end{tabular}

Telescope National Facility. Briefly, this array consists of six, 22-m telescopes arranged along an east-west baseline spanning $6 \mathrm{~km}$. A variety of configurations (" $6 \mathrm{~A}$ ", " $6 \mathrm{C}$ " $\&$ " $6 \mathrm{D}$ ") was used, with minimum baseline separations ranging from 77 to $337 \mathrm{~m}$ and maximum separations of close to $6000 \mathrm{~m}$.

These re-measurements have provided positions with a typical accuracy (standard error) of around 0.6 arcsec in each coordinate for the stronger compact sources, flux densities at both 4800 and $8640 \mathrm{MHz}$, and structural information for the individual source components which is sufficient to outline the gross morphology of the sources.

Our observing method made brief "cut" observations of the target sources, each lasting about 45 seconds. Every source was observed 3 times: once approximately $4 \mathrm{hrs}$ east of transit, once near transit, and once approximately $4 \mathrm{hrs}$ west of transit. The profile resulting from each cut observation was reduced and displayed in real time, thus permitting data quality to be continuously monitored. When three cuts had been obtained at the three different hour angles, the profiles were reduced and combined to produce positions and fluxes for all source components.

At the present time, over 8,000 sources have been measured and good positional, flux and structural data has been obtained for 7345 components of 6603 sources.

\section{Optical Identifications}

The accurate positions resulting from our Compact Array observations have been used, together with the COSMOS digitised sky survey (Yentis et al., 1992), to obtain optical identifications for over 4000 of the $P M N$ radio sources (Tasker and Wright, 1993; Tasker, 1996). However, while the COSMOS identifications are useful for the stellar objects, they are unreliable for the faintest or extended sources. We have therefore also used direct inspection of the UK Schmidt SERC J plates for the fainter, complex objects and to calibrate the automatic identification process. In addition, we also cross-correlated the Compact Array positions with the Hubble Space Telescope Guide Star Catalogue (GSC) to identify the brightest objects which 
may be saturated or unreliable on the COSMOS database.

The fainter $15<\mathrm{B}<20.5$ optical counterparts to our Compact Array Sample were determined using an automated procedure which interrogated the COSMOS database. The optical identifications were made without regard to optical colour or morphology, and were based solely on coincidence of the radio and optical positions. Identifications were claimed if they were better then $95 \%$ reliable, as estimated from the combined error in position and the density of background objects (which varied with position on the sky).

All the COSMOS identifications were confirmed using visual inspection of a set of "postage stamp" images extracted from the Hubble Space Telescope Digital Sky Survey (DSS) CD-ROMs. The reliability of our identification procedure was also calibrated using a control sample of around 500 radio sources which were also identified using original SERC J plate material.

Approximately $40 \%$ of all objects in our Compact Array Sample were identified using the COSMOS database alone. And we estimate that a further $5 \%$ would be identified if detailed inspection of the SERC J plates were possible for all the radio sources in the sample

Our second identification program used the Hubble GSC to determine the brighter $(9<\mathrm{B}<15)$ counterparts to the PMN Compact Array sample. We used the GSC to identify all sources within a distance of 10 arcsec from the radio centroid. The reliability of an identification was assessed from a histogram of radio-optical separations. Any candidate identification was also confirmed using the postage stamp images extracted from the DSS images around the relevant PMN survey position. Using this technique, a total of 220 highly-significant, bright identifications were made.

The principal result of the identification work has been an homogeneous and complete sample of over 4000 extragalactic radio sources with reliable optical identifications.

\section{Research Projects}

Based on the identification work described in the previous section, we are presently engaged in three major astrophysical projects.

The first is using the identifications from the Compact Array radio sample to search for southern gravitational lens candidates. At present, all but a few of the confirmed gravitational lenses lie north of the celestial equator. Since it is very plausible that similar numbers should lie in the south, the dearth of southern confirmations must clearly reflect the lack of reliably identified southern candidates. Our project aims to correct this deficiency. 
Secondly, we have compiled an unbiased list of around 30 GPS radio sources, using the fluxes measured in our Compact Array programme together with fluxes from other data sources, where available. We have also obtained optical spectra for almost all of the objects in this sample using the Anglo-Australian 4-m Telescope. These spectra cover the wavelength range $3300-10,000 \AA$ and include objects as faint as 21 magnitude (B). The principal goal of this project is to test the conventional "wisdom" that GPS sources are preferentially found at the highest redshifts.

Finally, the aim of our third project was to search for optically very bright - but radio faint - QSO candidates which may have previously escaped detection. To do this, we have obtained optical spectroscopy for the 220 optically-bright identifications mentioned in the previous section. These spectra were obtained using the 1.9-m Mount Stromlo Observatory telescope of the Australian National University. At present, we report that no new, bright QSOs have been identified, although an appreciable number of new ultra-compact galaxies and planetary nebulae have been found.

\section{Conclusions}

Based on the PMN Southern Survey $\left(-87^{\circ}<\delta<-37^{\circ}\right)$ we have described a programme which has obtained accurate positions, fluxes at 4800 and $8600 \mathrm{MHz}$ and structure for over 6600 radio sources using the Australia Telescope Compact Array.

Using these positions we have optically identified over 4000 objects using a mixture of digital and conventional techniques. Furthermore, we have assessed the relative reliability of these two methods.

Finally we are using the identified sources to search for southern gravitational lens candidates; to compile, and obtain optical spectroscopy for, an unbiased list of GPS radio sources; and to search for very bright QSO candidates that may have previously escaped detection.

\section{References}

Gregory, P.C. \& Condon, J.J. 1991, ApJS, 75, 1011

Griffith, M.R., \& Wright A.E. 1993, $A J, 105,1666$

Tasker, N. \& Wright, A.E., 1993, Proc. ASA, 10, 4, 320

Tasker, N., Wright, A.E., Griffith, M.R., \& Condon, J.J.1994, AJ, 107, 2115

Tasker, N., 1996, PhD thesis, In preparation

Wright, A.E. \& Otrupcek, R., eds. 1990, ATNF, "PKSCAT90 - the southern radio database", (Available via "anonymous FTP" from ftp.atnf.csiro.au under the subdirectory /pub/data/pkscat90)

Wright, A.E., Griffith, M.R., Burke, B.F., \& Ekers, R.D. 1994, ApJS, 91, 111

Yentis et al., 1992, "The COSMOS/UKST Catalogue of the Southern Sky", in Digitised Optical Sky Surveys, Astrophysics \& Space Science Library, 174, 67 